This item was submitted to Loughborough's Research Repository by the author.

Items in Figshare are protected by copyright, with all rights reserved, unless otherwise indicated.

\title{
Polarons in complex oxides and molecular nanowires
}

PLEASE CITE THE PUBLISHED VERSION

LICENCE

CC BY-NC-ND 4.0

\section{REPOSITORY RECORD}

Alexandrov, A.S.. 2019. "Polarons in Complex Oxides and Molecular Nanowires". figshare. https://hdl.handle.net/2134/1165. 


\title{
POLARONS IN COMPLEX OXIDES AND MOLECULAR NANOWIRES
}

\author{
A. S. Alexandrov \\ Department of Physics, Loughborough University, \\ Loughborough, United Kingdom \\ a.s.alexandrov@lboro.ac.uk
}

\begin{abstract}
There is a growing understanding that transport properties of complex oxides and individual molecules are dominated by polaron physics. In superconducting oxides the long-range Fröhlich and short-range JahnTeller electron-phonon interactions bind carriers into real space pairs small bipolarons with surprisingly low mass but sufficient binding energy, while the long-range Coulomb repulsion keeps bipolarons apart preventing their clustering. The bipolaron theory numerically explains high Tc values without any fitting parameters and describes other key features of the cuprates. The same approach provides a new insite into the theory of transport through molecular nanowires and quantum dots (MQD). Attractive polaron-polaron correlations lead to a "switching" phenomenon in the current-voltage characteristics of MQD. The degenerate MQD with strong electron-vibron coupling has two stable current states (a volatile memory), which might be useful in molecular electronics.
\end{abstract}

Keywords: Polarons, bipolarons, superconductivity, molecular memory

\section{Introduction}

When the electron-phonon (e-ph) interaction energy $E_{p}$ is larger than their kinetic energy, electrons in the Bloch band are "dressed" by phonons. If phonon frequencies are very low, the local lattice deformation traps the electron even in a perfect crystal lattice. This self-trapping phenomenon was predicted by Landau [1]. It has been studied in greater detail by Pekar [2], Fröhlich [3], Feynman [4], Devreese [5] and other authors in the effective mass approximation, which leads to the so-called large or continuous polaron. The large polaron propagates through the lattice as a free electron but with an enhanced effective mass.

In the strong-coupling regime, $\lambda=E_{p} / D>1$, the finite bandwidth $2 D$ becomes important, so that the effective mass approximation can- 
not be applied. The electron is called a small or lattice polaron in this regime. The self-trapping is never "complete", that is any polaron can tunnel through the lattice. Only in the extreme adiabatic limit, when the phonon frequencies tend to zero, the self-trapping is complete, and the polaron motion is no longer translationally continuous. The main features of the small polaron were understood by Tjablikov [6], Yamashita and Kurosava [7], Sewell [8], Holstein [9] and his school [10, 11], Lang and Firsov [12], Eagles [13], and others and described in several review papers and textbooks [5, 14-18]. An exponential reduction of the bandwidth at large values of $\lambda$ and phonon side-bands are among those features.

The lattice deformation also strongly affects the interaction between electrons. At large distances polarons repel each other in ionic crystals, but their Coulomb repulsion is substantially reduced due to the ion polarization. Nevertheless two large polarons can be bound into a large bipolaron by an exchange interaction even with no additional e-ph interaction but the Fröhlich one [5].

When a short-range deformation potential and molecular-type (i.e. Jahn-Teller [19]) e-ph interactions are taken into account together with the Fröhlich interaction [20], they can overcome the Coulomb repulsion. The resulting interaction becomes attractive at a short distance of about a lattice constant. Then two small polarons easily form a bound state, i.e. a small bipolaron, because their band is narrow. Consideration of particular lattice structures shows that small bipolarons are mobile even when the electron-phonon coupling is strong and the bipolaron binding energy is large [20]. Hence the polaronic Fermi liquid transforms into a Bose liquid of double-charged carriers in the strong-coupling regime. The Bose-liquid is stable because bipolarons repel each other [20]. Here we encounter a novel electronic state of matter, a charged Bose liquid, qualitatively different from the normal Fermi-liquid and from the BCS superfluid.

Experimental evidence for an exceptionally strong electron-phonon interaction in high temperature superconductors is now overwhelming. As we discussed in detail elsewhere [21], the extension of the BCS theory towards the strong interaction between electrons and ion vibrations describes the phenomenon naturally. High temperature superconductivity exists in the crossover region of the electron-phonon interaction strength from the BCS-like to bipolaronic superconductivity as was predicted before [22], and explored in greater detail by many authors after the discovery [23].

Small polarons with their phonon side-bands and attractive correlations are quite feasible also in molecular nanowires and quantum dots 
(MQD) used as the "transmission lines" [24, 25] and active molecular elements $[26,27]$ in molecular-scale electronics [26]. It has been experimentally demonstrated that the low-bias conductance of molecules is dominated by resonant tunneling through coupled electronic and vibration levels [28]. Conductance peaks due to electron-vibron interactions has been seen in $\mathrm{C}_{60}$ [29]. Different aspects of the electron-phonon/vibron (e-ph) interaction effect on the tunneling through molecules and quantum dots (QD) have been studied by several authors [30-36]. In particular, Glazman and Shekhter, and later Wingreen et al.[30] presented the exact resonant-tunneling transmission probability fully taking into account the e-ph interaction on a nondegenerate resonant site. Phonons produced transmission side-bands but did not affect the integral transmission probability. Li, Chen and Zhou [31] studied the conductance of a double degenerate (due to spin) quantum dot with Coulomb repulsion and the e-ph interaction. Their numerical results also showed the sideband peaks and the main peak related to the Coulomb repulsion, which was decreased by the e-ph interaction. Kang [32] studied the boson (vibron) assisted transport through a double-degenerate QD coupled to two superconducting leads and found multiple peaks in the I-V curves, which originated from the singular BCS density of states and the phonon side-bands.

While a correlated transport through mesoscopic systems with repulsive electron-electron interactions received considerable interest in the past, and continues to be the focus of intense investigations [37], much less has been known about a role of attractive correlations in MQD. Recently we have proposed a negative- $U$ Hubbard model of a $d$-fold degenerate quantum dot [38]. We argued that the attractive electron correlations caused by a strong electron-phonon (vibron) interaction in the molecule, and/or by the valence fluctuations provide a molecular switching effect, when the current-voltage (I-V) characteristics show two branches with high and low current for the same voltage. The effect was observed in a few experimental studies with complex [27] and simple molecules [39].

Here we review the analytical theory of a correlated transport through a degenerate molecule quantum dot (MQD) fully taking into account both Coulomb and e-ph interactions [40]. We show that the phonon side-bands significantly modify the switching behavior of the I-V curves in comparison with the negative- $U$ Hubbard model [38]. Nevertheless, the switching effect is robust. It shows up when the effective interaction of polarons is attractive and the state of the dot is multiply degenerate, $d>2$. 


\section{Attractive correlations of small polarons}

Employing the canonical polaron formalism with a generic "FröhlichCoulomb" Hamiltonian, allows us explicitly calculate the effective attraction of small polarons [41]. The Hamiltonian includes the infinite-range Coulomb, $V_{c}$ and electron-phonon interactions. The implicitly present infinite on-site repulsion (Hubbard $U$ ) prohibits double occupancy and removes the need to distinguish the fermionic spin. Introducing spinless fermion operators $c_{\mathbf{n}}$ and phonon operators $d_{\mathbf{m} \nu}$, the Hamiltonian is written as

$$
\begin{aligned}
H= & \sum_{\mathbf{n} \neq \mathbf{n}^{\prime}} T\left(\mathbf{n}-\mathbf{n}^{\prime}\right) c_{\mathbf{n}}^{\dagger} c_{\mathbf{n}^{\prime}}+\sum_{\mathbf{n} \neq \mathbf{n}^{\prime}} V_{c}\left(\mathbf{n}-\mathbf{n}^{\prime}\right) c_{\mathbf{n}}^{\dagger} c_{\mathbf{n}} c_{\mathbf{n}^{\prime}}^{\dagger} c_{\mathbf{n}^{\prime}}+ \\
& \omega_{0} \sum_{\mathbf{n} \neq \mathbf{m}, \nu} g_{\nu}(\mathbf{m}-\mathbf{n})\left(\mathbf{e}_{\nu} \cdot \mathbf{e}_{\mathbf{m}-\mathbf{n}}\right) c_{\mathbf{n}}^{\dagger} c_{\mathbf{n}}\left(d_{\mathbf{m} \nu}^{\dagger}+d_{\mathbf{m} \nu}\right)+ \\
& \omega_{0} \sum_{\mathbf{m}, \nu}\left(d_{\mathbf{m} \nu}^{\dagger} d_{\mathbf{m} \nu}+\frac{1}{2}\right) .
\end{aligned}
$$

The e-ph term is written in real space, which is more convenient in working with complex lattices.

In general, the many-body model Eq.(1) is of considerable complexity. However, we are interested in the limit of the strong e-ph interaction. In this case, the kinetic energy is a perturbation and the model can be grossly simplified using the canonical transformation [12] in the Wannier representation for electrons and phonons,

$$
S=\sum_{\mathbf{m} \neq \mathbf{n}, \nu} g_{\nu}(\mathbf{m}-\mathbf{n})\left(\mathbf{e}_{\nu} \cdot \mathbf{e}_{\mathbf{m}-\mathbf{n}}\right) c_{\mathbf{n}}^{\dagger} c_{\mathbf{n}}\left(d_{\mathbf{m} \nu}^{\dagger}-d_{\mathbf{m} \nu}\right)
$$

The transformed Hamiltonian is

$$
\begin{aligned}
\tilde{H}= & e^{-S} H e^{S}=\sum_{\mathbf{n} \neq \mathbf{n}^{\prime}} \hat{\sigma}_{\mathbf{n n}^{\prime}} c_{\mathbf{n}}^{\dagger} c_{\mathbf{n}^{\prime}}+\omega_{0} \sum_{\mathbf{m} \alpha}\left(d_{\mathbf{m} \nu}^{\dagger} d_{\mathbf{m} \nu}+\frac{1}{2}\right)+ \\
& \sum_{\mathbf{n} \neq \mathbf{n}^{\prime}} v\left(\mathbf{n}-\mathbf{n}^{\prime}\right) c_{\mathbf{n}}^{\dagger} c_{\mathbf{n}} c_{\mathbf{n}^{\prime}}^{\dagger} c_{\mathbf{n}^{\prime}}-E_{p} \sum_{\mathbf{n}} c_{\mathbf{n}}^{\dagger} c_{\mathbf{n}} .
\end{aligned}
$$

The last term describes the energy gained by polarons due to e-ph interaction. $E_{p}$ is the familiar polaron level shift

$$
E_{p}=\omega_{0} \sum_{\mathbf{m} \nu} g_{\nu}^{2}(\mathbf{m}-\mathbf{n})\left(\mathbf{e}_{\nu} \cdot \mathbf{e}_{\mathbf{m}-\mathbf{n}}\right)^{2},
$$

which is independent of $\mathbf{n}$. The third term on the right-hand side in Eq.(2) is the polaron-polaron interaction:

$$
v\left(\mathbf{n}-\mathbf{n}^{\prime}\right)=V_{c}\left(\mathbf{n}-\mathbf{n}^{\prime}\right)-V_{p h}\left(\mathbf{n}-\mathbf{n}^{\prime}\right),
$$


where

$$
\begin{aligned}
V_{p h}\left(\mathbf{n}-\mathbf{n}^{\prime}\right)= & 2 \omega_{0} \sum_{\mathbf{m}, \nu} g_{\nu}(\mathbf{m}-\mathbf{n}) g_{\nu}\left(\mathbf{m}-\mathbf{n}^{\prime}\right) \times \\
& \left(\mathbf{e}_{\nu} \cdot \mathbf{e}_{\mathbf{m}-\mathbf{n}}\right)\left(\mathbf{e}_{\nu} \cdot \mathbf{e}_{\mathbf{m}-\mathbf{n}^{\prime}}\right) .
\end{aligned}
$$

The phonon-induced interaction $V_{p h}$ is due to displacements of common ions by two electrons. Finally, the transformed hopping operator $\hat{\sigma}_{\mathbf{n n}^{\prime}}$ in the first term in Eq.(2) is given by

$$
\begin{aligned}
\hat{\sigma}_{\mathbf{n n}^{\prime}}= & T\left(\mathbf{n}-\mathbf{n}^{\prime}\right) \exp \left[\sum _ { \mathbf { m } , \nu } \left[g_{\nu}(\mathbf{m}-\mathbf{n})\left(\mathbf{e}_{\nu} \cdot \mathbf{e}_{\mathbf{m}-\mathbf{n}}\right)\right.\right. \\
& \left.\left.-g_{\nu}\left(\mathbf{m}-\mathbf{n}^{\prime}\right)\left(\mathbf{e}_{\nu} \cdot \mathbf{e}_{\mathbf{m}-\mathbf{n}^{\prime}}\right)\right]\left(d_{\mathbf{m} \alpha}^{\dagger}-d_{\mathbf{m} \alpha}\right)\right] .
\end{aligned}
$$

This term is a perturbation at large $\lambda$. It is absent in an isolated MQD, modeled as a single degenerate atomic level, so that the canonical transformation solves the problem exactly for any number of electrons (see below). In a crystal the term allows for a bipolaron tunnelling and high temperature superconductivity [22]. In particular crystal structures like perovskites, the tunnneling appears already in the first order in $T(\mathbf{n})$, so that $\hat{\sigma}_{\mathbf{n n}^{\prime}}$ can be averaged over phonons. The result is

$$
\begin{aligned}
& t\left(\mathbf{n}-\mathbf{n}^{\prime}\right) \equiv\left\langle\left\langle\hat{\sigma}_{\mathbf{n} \mathbf{n}^{\prime}}\right\rangle\right\rangle_{p h}=T\left(\mathbf{n}-\mathbf{n}^{\prime}\right) \exp \left[-g^{2}\left(\mathbf{n}-\mathbf{n}^{\prime}\right)\right] \\
& g^{2}\left(\mathbf{n}-\mathbf{n}^{\prime}\right)= \sum_{\mathbf{m}, \nu} g_{\nu}(\mathbf{m}-\mathbf{n})\left(\mathbf{e}_{\nu} \cdot \mathbf{e}_{\mathbf{m}-\mathbf{n}}\right) \times \\
& {\left[g_{\nu}(\mathbf{m}-\mathbf{n})\left(\mathbf{e}_{\nu} \cdot \mathbf{e}_{\mathbf{m}-\mathbf{n}}\right)-g_{\nu}\left(\mathbf{m}-\mathbf{n}^{\prime}\right)\left(\mathbf{e}_{\nu} \cdot \mathbf{e}_{\left.\left.\mathbf{m}-\mathbf{n}^{\prime}\right)\right] .}\right.\right.}
\end{aligned}
$$

By comparing Eqs.(6) and Eqs.(3,4), the mass renormalization exponent can be expressed via $E_{p}$ and $V_{p h}$ as follows

$$
g^{2}\left(\mathbf{n}-\mathbf{n}^{\prime}\right)=\frac{1}{\omega_{0}}\left[E_{p}-\frac{1}{2} V_{p h}\left(\mathbf{n}-\mathbf{n}^{\prime}\right)\right] .
$$

When $V_{p h}$ is larger than $V_{c}$ the full interaction becomes negative and polarons form pairs. The real space representation allows us to elaborate more physics behind the lattice sums in Eq.(3) and Eq.(4). If a carrier (electron or hole) acts on an ion with a force $\mathbf{f}$, it displaces the ion by some vector $\mathbf{x}=\mathbf{f} / s$. Here $s$ is the ion's force constant. The total energy of the carrier-ion pair is $-\mathbf{f}^{2} /(2 s)$. This is precisely the summand in Eq.(3) expressed via dimensionless coupling constants. Now consider two carriers interacting with the same ion, see Fig.1a. The ion displacement 




(b)



Figure 1. The mechanism of polaron-polaron interaction. (a) Together, the two polarons (solid circles) deform the lattice more effectively than separately. An effective attraction occurs when the angle $\phi$ is less than $\pi / 2$. (b) A mixed situation. Ion 1 results in repulsion between two polarons while ion 2 results in attraction.

is $\mathbf{x}=\left(\mathbf{f}_{1}+\mathbf{f}_{2}\right) / s$ and the energy is $-\mathbf{f}_{1}^{2} /(2 s)-\mathbf{f}_{2}^{2} /(2 s)-\left(\mathbf{f}_{1} \cdot \mathbf{f}_{2}\right) / s$. Here the last term should be interpreted as an ion-mediated interaction between the two carriers. It depends on the scalar product of $\mathbf{f}_{1}$ and $\mathbf{f}_{2}$ and consequently on the relative positions of the carriers with respect to the ion. If the ion is an isotropic harmonic oscillator, as we assume here, then the following simple rule applies. If the angle $\phi$ between $\mathbf{f}_{1}$ and $\mathbf{f}_{2}$ is less than $\pi / 2$ the polaron-polaron interaction will be attractive, if otherwise it will be repulsive. In general, some ions will generate attraction, and some repulsion between polarons, Fig. 1 b.

The overall sign and magnitude of the interaction is given by the lattice sum in Eq.(4), the evaluation of which is elementary. One should also note that according to Eq.(7) an attractive interaction reduces the polaron mass (and consequently the bipolaron mass), while repulsive interaction enhances the mass.

\section{Steady current through MQD}

Let us now apply the polaron formalism to MQD [40]. Here bipolarons might not exist because of a finite lifetime of electrons on a molecule connected with the leads, but the attractive correlations could strongly modify the current-voltage characteristics. We employ the Landauertype expression for a steady current through a region of interacting elec- 


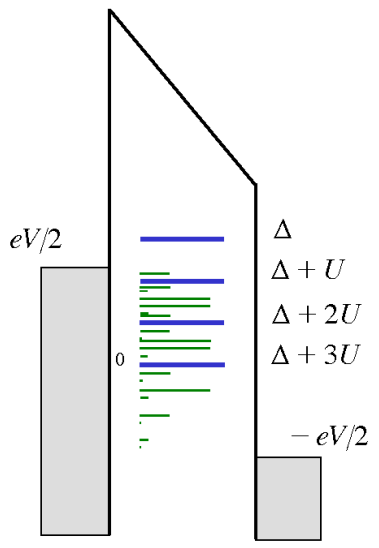

Figure 2. Schematic of the energy levels and phonon side-bands for molecular quantum dot under bias voltage $V(e V / 2 \Delta=0.75)$ with the coupling constant $\gamma^{2}=$ $11 / 13$. The level is assumed to be 4 -fold degenerate $(d=4)$ with energies $\Delta+r U$, $r=0, \ldots,(d-1)$ (thick bars). Thin bars show the vibron side-bands with the size of the bar proportional to the weight of the particular contribution in the density of states (see text) in the case of one vibron with frequency $\omega_{0} / \Delta=0.2$ at $T=0$. Only the bands in the energy window $(e V / 2,-e V / 2)$ (shown) will contribute to current at zero temperature.

trons, derived by Meir and Wingreen [42] as (in units $\hbar=k_{B}=1$ )

$$
I(V)=-\frac{e}{\pi} \int_{-\infty}^{\infty} d \omega\left[f_{1}(\omega)-f_{2}(\omega)\right] \operatorname{Im} \operatorname{Tr}\left[\hat{\Gamma}(\omega) \hat{G}^{R}(\omega)\right],
$$

where $f_{1(2)}(\omega)=\{\exp [(\omega+\Delta \mp e V / 2) / T]+1\}^{-1}, T$ is the temperature, $\Delta$ is the position of the lowest unoccupied molecular level with respect to the chemical potential. $\hat{\Gamma}(\omega)$ depends on the density of states (DOS) in the leads and on the hopping integrals connecting one-particle states in the left (1) and the right (2) leads with the states in MQD, Fig. 2.

This formula includes, by means of the Fourier transform of the full molecular retarded Green's function $(\mathrm{GF}), \hat{G}^{R}(\omega)$, the e-ph and Coulomb interactions inside the MQD and coupling to the leads. Since the leads are metallic, electron-electron and e-ph interactions in the leads, and interactions of electrons in the leads with electrons and phonons in the MQD can be neglected. We are interested in the tunneling near the conventional threshold, $e V=2 \Delta$, Fig.2, within a voltage range about an effective attractive potential $|U|$ caused by phonons/vibrons (see below).

The attractive energy is the difference of two large interactions, the Coulomb repulsion and the phonon mediated attraction, of the order 
of $1 \mathrm{eV}$ each. Hence, $|U|$ is of the order of a few tens of one eV. We neglect the energy dependence of $\hat{\Gamma}(\omega) \approx \Gamma$ on this scale, and assume that the coupling to the leads is weak, $\Gamma \ll|U|$. In this case $\hat{G}^{R}(\omega)$ does not depend on the leads. Moreover we assume that there is a complete set of one-particle molecular states $|\mu\rangle$, where $\hat{G}^{R}(\omega)$ is diagonal. With these assumptions we can reduce Eq.(8) to

$$
I(V)=I_{0} \int_{-\infty}^{\infty} d \omega\left[f_{1}(\omega)-f_{2}(\omega)\right] \rho(\omega),
$$

allowing for a transparent analysis of essential physics of the switching phenomenon. Here $I_{0}=e \Gamma$ and the molecular DOS, $\rho(\omega)$, is given by

$$
\rho(\omega)=-\frac{1}{\pi} \sum_{\mu} \operatorname{Im} \hat{G}_{\mu}^{R}(\omega),
$$

where $\hat{G}_{\mu}^{R}(\omega)$ is the Fourier transform of $\hat{G}_{\mu}^{R}(t)=-i \theta(t)\left\langle\left\{c_{\mu}(t), c_{\mu}^{\dagger}\right\}\right\rangle$, $\{\cdots, \cdots\}$ is the anticommutator, $c_{\mu}(t)=e^{i H t} c_{\mu} e^{-i H t}, \theta(t)=1$ for $t>0$ and zero otherwise.

We calculate $\rho(\omega)$ exactly ( see below) in the framework of the Hamiltonian, which includes both the Coulomb $U^{C}$ and e-ph interactions as

$$
\begin{aligned}
H= & \sum_{\mu} \varepsilon_{\mu} \hat{n}_{\mu}+\frac{1}{2} \sum_{\mu \neq \mu^{\prime}} U_{\mu \mu^{\prime}}^{C} \hat{n}_{\mu} \hat{n}_{\mu^{\prime}} \\
& +\sum_{\mu, q} \hat{n}_{\mu} \omega_{q}\left(\gamma_{\mu q} d_{q}+\text { H.c. }\right)+\sum_{q} \omega_{q}\left(d_{q}^{\dagger} d_{q}+1 / 2\right) .
\end{aligned}
$$

Here $\varepsilon_{\mu}$ are one-particle molecular energy levels, $\hat{n}_{\mu}=c_{\mu}^{\dagger} c_{\mu}$ the occupation number operators, $c_{\mu}$ and $d_{q}$ annihilates electrons and phonons, respectively, $\omega_{q}$ are the phonon (vibron) frequencies, and $\gamma_{\mu q}$ are e-ph coupling constants ( $q$ enumerates the vibron modes). This Hamiltonian conserves the occupation numbers of molecular states $\hat{n}_{\mu}$. Hence it is compatible with Eq.(9).

\section{MQD density of states}

We apply the canonical polaron unitary transformation $e^{S}$, as in Section 1 , integrating phonons out. The electron and phonon operators are transformed as

$$
\tilde{c}_{\mu}=c_{\mu} X_{\mu}
$$

and

$$
\tilde{d}_{q}=d_{q}-\sum_{\mu} \hat{n}_{\mu} \gamma_{\mu q}^{*}
$$


respectively. Here

$$
X_{\mu}=\exp \left[\sum_{q} \gamma_{\mu q} d_{q}-H . c .\right]
$$

The Lang-Firsov canonical transformation shifts ions to new equilibrium positions with no effect on the phonon frequencies. The diagonalization is exact in MQD:

$$
\tilde{H}=\sum_{i} \tilde{\varepsilon}_{\mu} \hat{n}_{\mu}+\sum_{q} \omega_{q}\left(d_{q}^{\dagger} d_{q}+1 / 2\right)+\frac{1}{2} \sum_{\mu \neq \mu^{\prime}} U_{\mu \mu^{\prime}} \hat{n}_{\mu} \hat{n}_{\mu^{\prime}},
$$

where

$$
U_{\mu \mu^{\prime}} \equiv U_{\mu \mu^{\prime}}^{C}-2 \sum_{q} \gamma_{\mu q}^{*} \gamma_{\mu^{\prime} q} \omega_{q}
$$

is the interaction of polarons comprising their interaction via molecular deformations (vibrons) and non-vibron (e.g. Coulomb repulsion) $U_{\mu \mu^{\prime}}^{C}$. To simplify the discussion, we shall assume, that the Coulomb integrals do not depend on the orbital index, i.e. $U_{\mu \mu^{\prime}}=U$.

The molecular energy levels are shifted by the polaron level-shift due to a deformation well created by polaron,

$$
\tilde{\varepsilon}_{\mu}=\varepsilon_{\mu}-\sum_{q}\left|\gamma_{\mu q}\right|^{2} \omega_{q}
$$

Applying the same transformation in the retarded GF we obtain

$$
\begin{aligned}
G_{\mu}^{R}(t)= & -i \theta(t)\left\langle\left\{c_{\mu}(t) X_{\mu}(t), c_{\mu}^{\dagger} X_{\mu}^{\dagger}\right\}\right\rangle \\
= & -i \theta(t)\left[\left\langle c_{\mu}(t) c_{\mu}^{\dagger}\right\rangle\left\langle X_{\mu}(t) X_{\mu}^{\dagger}\right\rangle\right. \\
& \left.+\left\langle c_{\mu}^{\dagger} c_{\mu}(t)\right\rangle\left\langle X_{\mu}^{\dagger} X_{\mu}(t)\right\rangle\right],
\end{aligned}
$$

where now electron and phonon operators are averaged over the quantum state of the transformed Hamiltonian $\tilde{H}$. There is no coupling between polarons and vibrons in the transformed Hamiltonian, so that

$$
\begin{aligned}
& \left\langle X_{\mu}(t) X_{\mu}^{\dagger}\right\rangle \\
= & \exp \left[\sum_{q} \frac{\left|\gamma_{\mu q}\right|^{2}}{\sinh \frac{\beta \omega_{q}}{2}}\left[\cos \left(\omega t+i \frac{\beta \omega_{q}}{2}\right)-\cosh \frac{\beta \omega_{q}}{2}\right]\right],
\end{aligned}
$$

where $\beta=1 / T$, and $\left\langle X_{\mu}^{\dagger} X_{\mu}(t)\right\rangle=\left\langle X_{\mu}(t) X_{\mu}^{\dagger}\right\rangle^{*}$. 
Next, we introduce the $N$-particle GFs, which will necessarily appear in the equations of motion for $\left\langle c_{\mu}(t) c_{\mu}^{\dagger}\right\rangle$, as

$$
G_{\mu}^{(N,+)}(t) \equiv-i \theta(t) \sum_{\mu_{1} \neq \mu_{2} \neq \ldots \mu}\left\langle c_{\mu}(t) c_{\mu}^{\dagger} \prod_{i=1}^{N-1} \hat{n}_{\mu_{i}}\right\rangle,
$$

and

$$
G_{\mu}^{(N,-)}(t) \equiv-i \theta(t) \sum_{\mu_{1} \neq \mu_{2} \neq \ldots \mu}\left\langle c_{\mu}^{\dagger} c_{\mu}(t) \prod_{i=1}^{N-1} \hat{n}_{\mu_{i}}\right\rangle .
$$

Then, using the equation of motion for the Heisenberg polaron operators, we derive the following equations for the $N$-particle GFs,

$$
\begin{aligned}
i \frac{d G_{\mu}^{(N,+)}(t)}{d t}= & \delta(t)\left(1-n_{\mu}\right) \sum_{\mu_{1} \neq \mu_{2} \neq \ldots \mu} \prod_{i=1}^{N-1} n_{\mu_{i}} \\
& +\left[\tilde{\varepsilon}_{\mu}+(N-1) U\right] G_{\mu}^{(N,+)}(t)+U G_{\mu}^{(N+1,+)}(t),
\end{aligned}
$$

and

$$
\begin{aligned}
i \frac{d G_{\mu}^{(N,-)}(t)}{d t}= & \delta(t) n_{\mu} \sum_{\mu_{1} \neq \mu_{2} \neq \ldots \mu} \prod_{i=1}^{N-1} n_{\mu_{i}} \\
& +\left[\tilde{\varepsilon}_{\mu}+(N-1) U\right] G_{\mu}^{(N,-)}(t)+U G_{\mu}^{(N+1,-)}(t)
\end{aligned}
$$

where $n_{\mu}=\left\langle c_{\mu}^{\dagger} c_{\mu}\right\rangle$ is the expectation number of electrons on the molecular level $\mu$.

We readily solve this set of coupled equations for MQD with one $d$-fold degenerate energy level and with the e-ph coupling $\gamma_{\mu q}=\gamma_{q}$, which does not break the degeneracy. Assuming that $n_{\mu}=n$, Fourier transformation of the set yields for $N=1$

$$
\begin{gathered}
G_{\mu}^{(1,+)}(\omega)=(1-n) \sum_{r=0}^{d-1} \frac{Z_{r}(n)}{\omega-r U+i \delta} \\
G_{\mu}^{(1,-)}(\omega)=n \sum_{r=0}^{d-1} \frac{Z_{r}(n)}{\omega-r U+i \delta}
\end{gathered}
$$

where $\delta=+0$, and

$$
Z_{r}(n)=\frac{(d-1) !}{r !(d-1-r) !} n^{r}(1-n)^{d-1-r} .
$$


In approximation, where we retain a coupling to a single mode with the characteristic frequency $\omega_{0}$ and $\gamma_{q} \equiv \gamma$, the molecular DOS is readily found as an imaginary part of the Fourier transform of Eq.(17) using Eqs.(23,24) and Eq.(18):

$$
\begin{aligned}
& \rho(\omega)=\mathcal{Z} d \sum_{r=0}^{d-1} Z_{r}(n) \sum_{l=0}^{\infty} I_{l}(\xi) \\
& \times\left[e^{\frac{\beta \omega_{0} l}{2}}\left[(1-n) \delta\left(\omega-r U-l \omega_{0}\right)+n \delta\left(\omega-r U+l \omega_{0}\right)\right]\right. \\
& +\left(1-\delta_{l 0}\right) e^{-\frac{\beta \omega_{0} l}{2}}\left[n \delta\left(\omega-r U-l \omega_{0}\right)\right. \\
& \left.\left.+(1-n) \delta\left(\omega-r U+l \omega_{0}\right)\right]\right]
\end{aligned}
$$

where

$$
\mathcal{Z}=\exp \left[-\sum_{\mathbf{q}}\left|\gamma_{q}\right|^{2} \operatorname{coth} \frac{\beta \omega_{q}}{2}\right],
$$

$\xi=|\gamma|^{2} / \sinh \frac{\beta \omega_{0}}{2}, I_{l}(\xi)$ is the modified Bessel function, and $\delta_{l k}$ is the Kroneker symbol. The important feature of the DOS, Eq.(26), is its nonlinear dependence on the occupation number $n$, which leads to the switching effect and hysteresis in the I-V characteristics for $d>2$, as will be shown below. It contains full information about all possible correlation and inelastic effects in transport, in particular, all the vibronassisted tunneling processes and phonon sidebands, and describes the renormalization of hopping to the leads.

\section{Nonlinear rate equation and switching}

Generally, the electron density $n_{\mu}$ obeys an infinite set of rate equations for many-particle GFs which can be derived in the framework of a tunneling Hamiltonian including correlations [38]. In the case of MQD only weakly coupled with leads one can apply the Fermi-Dirac golden rule to obtain an equation for $n$. Equating incoming and outgoing numbers of electrons in MQD per unit time we obtain the self-consistent equation for the level occupation $n$ as

$$
\begin{aligned}
& (1-n) \int_{-\infty}^{\infty} d \omega\left\{\Gamma_{1} f_{1}(\omega)+\Gamma_{2} f_{2}(\omega)\right\} \rho(\omega) \\
& -n \int_{-\infty}^{\infty} d \omega\left\{\Gamma_{1}\left[1-f_{1}(\omega)\right]+\Gamma_{2}\left[1-f_{2}(\omega)\right]\right\} \rho(\omega)=0
\end{aligned}
$$

where $\Gamma_{1(2)}$ are the transition rates from left (right) leads to MQD. Taking into account that $\int_{-\infty}^{\infty} \rho(\omega)=d$, Eq.(28) for the symmetric leads, 
$\Gamma_{1}=\Gamma_{2}$, reduces to

$$
2 n d=\int d \omega \rho(\omega)\left(f_{1}+f_{2}\right)
$$

which automatically satisfies $0 \leq n \leq 1$. Explicitly, the self-consistent equation for the occupation number is

$$
n=\frac{1}{2} \sum_{r=0}^{d-1} Z_{r}(n)\left[n a_{r}+(1-n) b_{r}\right],
$$

where

$$
\begin{aligned}
a_{r}= & \mathcal{Z} \sum_{l=0}^{\infty} I_{l}(\xi)\left(e^{\frac{\beta \omega_{0} l}{2}}\left[f_{1}\left(r U-l \omega_{0}\right)+f_{2}\left(r U-l \omega_{0}\right)\right]\right. \\
& \left.+\left(1-\delta_{l 0}\right) e^{-\frac{\beta \omega_{0} l}{2}}\left[f_{1}\left(r U+l \omega_{0}\right)+f_{2}\left(r U+l \omega_{0}\right)\right]\right), \\
b_{r}= & \mathcal{Z} \sum_{l=0}^{\infty} I_{l}(\xi)\left(e^{\frac{\beta \omega_{0} l}{2}}\left[f_{1}\left(r U+l \omega_{0}\right)+f_{2}\left(r U+l \omega_{0}\right)\right]\right. \\
& \left.+\left(1-\delta_{l 0}\right) e^{-\frac{\beta \omega_{0} l}{2}}\left[f_{1}\left(r U-l \omega_{0}\right)+f_{2}\left(r U-l \omega_{0}\right)\right]\right) .
\end{aligned}
$$

The current is expressed as

$$
j \equiv \frac{I(V)}{d I_{0}}=\sum_{r=0}^{d-1} Z_{r}(n)\left[n a_{r}^{\prime}+(1-n) b_{r}^{\prime}\right],
$$

where

$$
\begin{aligned}
a_{r}^{\prime}= & \mathcal{Z} \sum_{l=0}^{\infty} I_{l}(\xi)\left(e^{\frac{\beta \omega_{0} l}{2}}\left[f_{1}\left(r U-l \omega_{0}\right)-f_{2}\left(r U-l \omega_{0}\right)\right]\right. \\
& \left.+\left(1-\delta_{l 0}\right) e^{-\frac{\beta \omega_{0} l}{2}}\left[f_{1}\left(r U+l \omega_{0}\right)-f_{2}\left(r U+l \omega_{0}\right)\right]\right), \\
b_{r}^{\prime}= & \mathcal{Z} \sum_{l=0}^{\infty} I_{l}(\xi)\left(e^{\frac{\beta \omega_{0} l}{2}}\left[f_{1}\left(r U+l \omega_{0}\right)-f_{2}\left(r U+l \omega_{0}\right)\right]\right. \\
& \left.+\left(1-\delta_{l 0}\right) e^{-\frac{\beta \omega_{0} l}{2}}\left[f_{1}\left(r U-l \omega_{0}\right)-f_{2}\left(r U-l \omega_{0}\right)\right]\right) .
\end{aligned}
$$

There is only one physical $(0<n<0.5)$ solution of the rate equation (30) and no switching for a nondegenerate, $d=1$, and doubledegenerate, $d=2$, MQDs. However, the switching appears for $d>2$. 




Figure 3. The bistable I-V curves for tunneling through molecular quantum dot (Fig. 2) with the electron-vibron coupling constant $\gamma^{2}=11 / 13$ and $\omega_{0} / \Delta=0.2$. The up arrows show that the current picks up at some voltage when it is biased, and then drops at lower voltage when the bias is being reduced. The bias dependence of current basically repeats the shape of the level occupation $n$ (right column). Steps on the curve correspond to the changing population of the phonon side-bands, which are shown in Fig. 2.

For example, for $d=4$ the rate equation is of the fourth power in $n$,

$$
\begin{aligned}
2 n= & (1-n)^{3}\left[n a_{0}+(1-n) b_{0}\right] \\
& +3 n(1-n)^{2}\left[n a_{1}+(1-n) b_{1}\right] \\
& +3 n^{2}(1-n)\left[n a_{2}+(1-n) b_{2}\right] \\
& +n^{3}\left[n a_{3}+(1-n) b_{3}\right] .
\end{aligned}
$$

Differently from the non-degenerate or double-degenerate MQD, the rate equation (36) for $d=4$ has two stable physical roots in a certain voltage range and the current-voltage characteristics show a hysteretic behavior. We show the numerical results for $\omega_{0}=0.2$ (in units of $\Delta$, as all the energies in the problem) and $U^{C}=0$ for the coupling constant, $\gamma^{2}=11 / 13$ in Fig. 3. This case formally corresponds to a negative 
Hubbard $U=-2 \gamma^{2} \omega_{0} \approx-0.4$ (we selected those values of $\gamma^{2}$ to avoid accidental commensurability of the correlated levels separated by $U$ and the phonon side-bands). The threshold for the onset of bistability appears at a voltage bias $e V / 2 \Delta=0.86$ for $\gamma^{2}=11 / 13$ and $\left.\omega_{0}=0.2\right)$. The inelastic tunneling processes through the level, accompanied by emission/absorption of the vibrons, manifest themselves as steps on the I-V curve, Figs. 3. Those steps are generated by the phonon side-bands originating from correlated levels in the dot with the energies $\Delta, \Delta+U$, $\ldots, \Delta+(d-1) U$. Since $\omega_{0}$ is not generally commensurate with $U$, we obtain quite irregular picture of the steps in I-V curves. The bistability region shrinks down with temperature.

In conclusion, we have reviewed the multi-polaron theory of tunneling through a molecular quantum dot (MQD) taking phonon side-bands and attractive polaron correlations into account. The degenerate MQD with strong electron-vibron coupling shows a hysteretic volatile memory if the degeneracy of the molecular level is larger than two, $d>2$. The hysteretic behavior strongly depends on the electron-vibron coupling and characteristic vibron frequencies. The current bistability vanishes above some critical temperature. It would be very interesting to look for an experimental realization of the model, possibly in a system containing a certain conjugated central part, which exhibits the attractive correlations of carriers with large degeneracy $d>2$. Interesting candidate systems are $\mathrm{C}_{60}$ molecule $(d=6)$ where the electron-phonon interaction is strong [29], short nanotubes or other fullerenes $(d \gg 1)$, and

mixed-valence molecular complexes. Switching should be fast, $10^{-13} \mathrm{~s}$ or faster.

This work has been supported by DARPA, and by the Leverhulme Trust (UK).

\section{References}

[1] Landau L D 1933 J. Physics (USSR) 3664

[2] Pekar S I 1946 Zh. Eksp. Teor. Fiz. 16335

[3] Fröhlich H 1954 Adv. Phys. 3325

[4] Feynman R P 19551955 Phys. Rev. 97660

[5] Devreese J T 1996 in Encyclopedia of Applied Physics, vol. 14, p. 383 (VCH Publishers) and references therein

[6] Tjablikov S V 1952 Zh.Eksp.Teor.Fiz. 23381

[7] Yamashita J and T. Kurosawa 1958 J. Phys. Chem. Solids 534

[8] Sewell G L 1958 Phil. Mag. 31361

[9] Holstein T 1959 Ann. Phys. 8 325; ibid 343

[10] Friedman L and Holstein T 1963 Ann. Phys 21494 
[11] Emin D and Holstein T 1969 Ann. Phys 53439

[12] Lang I G and Firsov Yu A 1962 Zh. Eksp. Teor. Fiz. 43 1843; 1963 Sov. Phys. JETP 161301

[13] Eagles D M 1963 Phys. Rev. 130 1381; 1969 Phys. Rev. 181 1278; 1969 Phys. Rev. 186456

[14] Appel J 1968 in Solid State Physics 21 (eds. Seitz F, Turnbull D and Ehrenreich H, Academic Press)

[15] Firsov Yu A (ed) 1975 Polarons (Moscow: Nauka)

[16] Böttger H and Bryksin V V 1985 Hopping Conduction in Solids (Berlin: Academie-Verlag)

[17] Mahan G D 1990 Many Particle Physics (New York: Plenum Press)

[18] Alexandrov A S and Mott N F 1995 Polarons and Bipolarons (Singapore: World Scientific)

[19] Müller K A 2002 Physica Scripta T102 39, and references therein

[20] Alexandrov A S 1996 Phys. Rev. B53 2863

[21] Alexandrov A S 2003 Theory of Superconductivity: From Weak to Strong Coupling (Bristol and Philadelphia: IoP Publishing)

[22] Alexandrov A S 1983 Zh. Fiz. Khim. 57273 ; 1983 Russ. J. Phys. Chem. 57 167; 1998 Models and Phenomenology for Conventional and High-temperature Superconductivity (Course CXXXVI of the Intenational School of Physics 'Enrico Fermi', eds. G. Iadonisi, J.R. Schrieffer and M.L. Chiofalo, Amsterdam: IOS Press), p. 309

[23] see contributions in 1995 Polarons and Bipolarons in High-T $T_{c}$ Superconductors and Related Materials (eds. Salje E K H, Alexandrov A S and Liang W Y, Cambridge: Cambridge University Press), and in 1995 Anharmonic properties of High Tc cuprates (eds. Mihailovic D, Ruani G, Kaldis E and Muller K A, Singapore: World Scientific)

[24] Lehn J-M 1990 Angew. Chem. Int. Ed. Engl. 291304

[25] Tour J M 2000 Acc. Chem. Res. 33 791; Tour J M et al. 1995 J. Am. Chem. Soc. 1179529

[26] Aviram A and Ratner M 1998 Eds. Molecular Electronics: Science and Technology (Ann. N.Y. Acad. Sci., New York)

[27] Collier C P et al. 1999 Science 285391 ; Chen J et al. 1999 Science 286 1550; D.I. Gittins D I et al. 2000 Nature (London) 408677 ; He H X, Tao T J, Nagahara L A, Amlani I and Tsui R (unpublished).

[28] Zhitenev N B, Meng H, and Bao Z 2002 Phys. Rev. Lett. 88226801

[29] Park J, Pasupathy A N, Goldsmith J I, Chang C, Yaish Y, Retta J R, Rinkoski M, Sethna J P, Abruña H D, McEuen P L, and Ralph D C 2000 Nature (London) 417722

[30] Glazman L I and Shekhter R I 1987 Zh. Eksp. Teor. Fiz. 94292 [Sov. Phys. JETP 67, 163 (1988)]; Wingreen N S, Jacobsen K W, and Wilkins J W 1989 Phys. Rev. B 1711834

[31] Xi Li, Chen H, and Zhou S 1995 Phys. Rev. B52 12202

[32] Kang K 1998 Phys. Rev. B57, 11891 
[33] Ermakov V N 2000 Physica E8 99

[34] Di Ventra M, Kim S-G, Pantelides S T, and Lang N D 2001 Phys. Rev. Lett. 86 288

[35] Ness N, Shevlin S A, and Fisher A J 2001 Phys. Rev. B 63125422

[36] Lundin U and McKenzie R H 2002 Phys. Rev. B 66, 075303

[37] see this volume

[38] Alexandrov A S, Bratkovsky A M, and Williams R S 2003 Phys. Rev. B 67 075301

[39] Stewart D et al. (unpublished).

[40] Alexandrov A S and Bratkovsky A M 2003 Phys. Rev. B 67235312

[41] Alexandrov A S and Kornilovitch P E 2002 J. Phys.: Condens. Matter 145337

[42] Meir Y and Wingreen N S 1992 Phys. Rev. Lett. 682512 